\title{
O ENFERMEIRO NA VIGILÂNCIA EPIDEMIOLÓGICA NO MUNICÍPIO DE RIBEIRÃO PRETO 1988-1996*
}

\author{
Cesar Eduardo Pedersoli** \\ Elisângela Antonialli*** \\ Tereza Cristina Scatena Vila****
}

PEDERSOLI, C.E.; ANTONIALLI, E.; VILA, T.C.S. O enfermeiro na vigilância epidemiológica no município de

Ribeirão Preto 1988-1996. Rev. latino-am. enfermagem, Ribeirão Preto, v. 6, n. 5, p. 99-105, dezembro 1998.

O enfermeiro é inserido nas equipes de Vigilância Epidemiológica (VE) da Secretaria Municipal de Saúde de Ribeirão Preto no processo de municipalização da saúde de 1988 a 1996. A abordagem qualitativa é utilizada sendo os dados obtidos através de fontes documentais e bibliográficas e entrevistas semi-estruturadas. O profissional que predomina na equipe da VE é o enfermeiro, fundamentando sua prática na epidemiologia e na gerência, e suas ações se voltam para o controle representado pelas notificações e visitas e para a prevenção, representada pela imunização.

UNITERMOS: enfermagem de saúde pública, vigilância epidemiológica

\section{INTRODUÇÃO}

Esta investigação constitui-se em um estudo sobre a configuração da prática de enfermagem no âmbito da Vigilância Epidemiológica (VE) na Secretaria Municipal de Saúde de Ribeirão Preto, tendo por base o processo de municipalização da saúde nas décadas de 80 e 90.

A perspectiva de descrever o trabalho de enfermagem na VE como uma ação de saúde coletiva, inserida e articulada à organização dos serviços de saúde, emerge face ao processo de descentralização das ações de saúde no final da década de 80 . Período marcado por reformas no setor saúde, no âmbito da Constituição e Legislação Brasileira, caracterizadas por disputas pela hegemonia de modelos de organização e distribuição de serviços de saúde e pela descentralização da gestão e planejamento. Neste período do estudo viriam a se constituir equipes de saúde pública (médicos sanitaristas e enfermeiras predominantemente) que vão implantar e implementar o Sistema de Vigilância Epidemiológica (SVE) no município de Ribeirão Preto, repensando suas formas de organização, redefinindo competências, estratégias de intervenção e fluxo de informações.

O interesse neste eixo de investigação que estuda a enfermagem inserida na organização de serviços de saúde tem por finalidade não apenas as questões operacionais, relativas à conformação dos serviços de saúde, mas abordar as formas e alternativas de organização destes, ampliando o entendimento das práticas de saúde coletiva.

A atual legislação (Lei 8080) define a VE como "um conjunto de ações que proporcionam o conhecimento, a detecção e prevenção de qualquer mudança nos fatores determinantes e condicionantes de saúde individual e coletiva, com a finalidade de recomendar e adotar as medidas de prevenção e controle das doenças ou agravos."

Vislumbra-se, no município, uma tendência de se avançar rumo ao processo de descentralização em nível local, implantando estratégias que possibilitem maior autonomia e responsabilidade aos níveis mais periféricos. Esta proposta encaminha possibilidades de reestruturação dos serviços de saúde voltados para a atenção primária, incorporando um novo modelo de assistência.

Com a descentralização dos serviços de saúde pretende-se tornar possível a integração sanitária, ou seja, prestar serviços de saúde para a recuperação da saúde individual, bem como organizar medidas de ação coletiva

\footnotetext{
* Insere-se no projeto integrado de Pesquisa intitulado "O trabalho de enfermagem na organização das práticas de saúde"

** Aluno de Enfermagem da EERP-USP, bolsista de Iniciação Científica Fapesp

*** Aluna de Enfermagem da EERP-USP, bolsista de Iniciação científica - PIBIC - CNPq

**** Professora do Departamento de Enfermagem Materno-Infantil e Saúde Pública da Escola de Enfermagem de Ribeirão Preto da Universidade de São Paulo
} 
para o controle, prevenção e promoção da saúde da população (MENDES, 1993).

Essas mudanças na gestão do sistema de saúde também têm produzido modificações nos vários momentos do processo de trabalho em saúde, seja nos objetivos, finalidade, instrumentos e meios de trabalho, seja na introdução de novos agentes para operar essas práticas.

Esta investigação justifica-se pela necessidade de compreender as especificidades e a natureza do trabalho de enfermagem na Vigilância Epidemiológica, enquanto área de conhecimento da saúde pública, no processo de consolidação do Sistema Único de Saúde(SUS).

\section{OBJETIVOS}

O objetivo geral desta investigação é analisar a inserção do trabalho da enfermeira em uma área específica de trabalho - o Departamento de Vigilância Epidemiológica da Secretaria Municipal de Saúde de Ribeirão Preto, São Paulo, face ao processo de Municipalização da Saúde.

Objetivos específicos:

Identificar o quadro de profissionais que atuam no Departamento de Vigilância Epidemiológica da Secretaria Municipal de Saúde de Ribeirão Preto, caracterizando o quantitativo de enfermeiros e sua representatividade em relação aos outros profissionais que aí atuam (1988 - 1995);

Identificar e analisar os diferentes períodos em que o profissional enfermeiro foi inserido na $\mathrm{VE}$ a fim de evidenciar a natureza deste trabalho e as transformações na política de saúde do município de Ribeirão Preto.

\section{Quadro teórico: a atenção à saúde na dimensão coletiva}

Neste estudo utilizar-se-á as idéias e conceitos desenvolvidos por diferentes autores a fim de nortear o olhar sobre o trabalho em saúde coletiva e, mais especificamente, o trabalho de enfermagem na V.E.

A Epidemiologia segundo MENDES GONÇALVES (1986), ao tomar a saúde e a doença na sua dimensão coletiva como objeto de conhecimento, é capaz de produzir para a prática um saber dotado das mesmas características: não é nunca o caso individual de doença que lhe interessa, a não ser por seu significado indicativo de um conjunto. Por conceber esse conjunto desenvolve instrumentos para sua apreensão, indicadores de freqüência que podem levar à construção de quadros de descrição do estado de doença no coletivo.
A epidemiologia tornou-se, efetivamente, um instrumental tecnológico aplicado ao campo da saúde pública e indispensável para os trabalhadores de saúde que atuam em todos os níveis do Sistema Único de Saúde - SUS na reorganização da assistência pois, praticamente, toda atividade neste campo vem sendo permeada por este saber. Dentre as atividades destacam-se: -diagnóstico de saúde em nível local, regional ou central; -planejamento e avaliação das ações de saúde coletiva; -suporte às ações de intervenção, controle ou erradicação de doenças; definição das prioridades regionais e das políticas de saúde para o país. (ROUQUAYROL \& PONTES, 1994).

PAIM (1993) apresenta a noção de Distrito Sanitário ( DS) como um conceito estratégico para a reforma sanitária, entendido em termos de teoria da organização da assistência, enquanto modelo assistencial para atenção, prestação de saúde a uma dada população, onde busca combinações de tecnologias a serem acionadas em função de problemas de saúde identificados dentro de um dado perfil epidemiológico de uma população. Requer mais um trabalho epidemiologicamente orientado na perspectiva de uma atenção à saúde na sua dimensão coletiva do que um trabalho clinicamente dirigido para o cuidado médico individual.

Entretanto, CAMPOS (1997) amplia o âmbito de análise das práticas de saúde pública, apontando seus limites de atuação e perspectivas de reorganização, quando afirma que, na prática, há deficiências na construção das políticas de controle.

Para ampliar esta prática é necessário constituir equipes especializadas em saúde pública a fim de recuperar a capacidade operacional da área para fazer diagnósticos e planejar a atuação do SUS, de modo a intervir em cadeias de determinação com objetivo precípuo de produzir saúde.

\section{METODOLOGIA}

\section{O campo da pesquisa}

A investigação foi realizada no município de Ribeirão Preto, tomando como locus o Departamento de Vigilância Epidemiológica da Secretaria Municipal de Saúde que articula-se à rede hospitalar e ambulatorial. A rede básica de saúde é composta por 32 Unidades de Saúde, sendo 26 Unidades Básicas de Saúde e 5 Unidades Básicas e Distritais de Saúde, oferecendo atendimento básico e especializado, além de serviços ambulatoriais dos hospitais conveniados e contratados. 


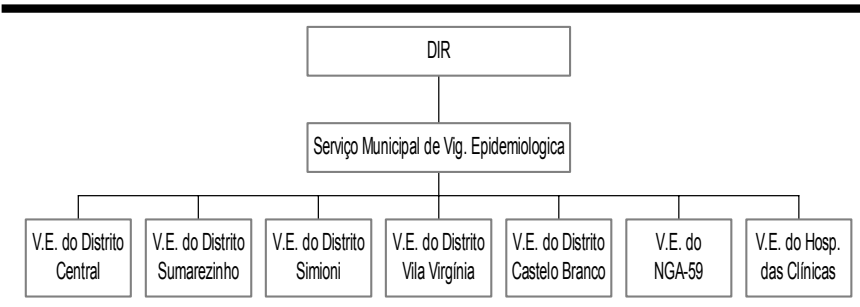

Figura 1 - Distribuição organizacional do serviço de vigilância epidemiológica do município

Encontra-se em gestão semi-plena $* * * * *$ desde o final de 1994, período do estudo, assumindo a gestão plena do sistema de saúde a partir de 1998.

\section{Coleta de dados}

Numa primeira etapa, a fim de reconstituir o período em estudo através de fontes bibliográficas e documentais, foram analisados documentos oficiais da Secretaria Municipal da Saúde e da Secretaria Estadual da Saúde.

Posteriormente foram selecionados os agentes a serem entrevistados, num total de 8 (oito) enfermeiras do Departamento de Vigilância Epidemiológica da Secretaria Municipal de Saúde de Ribeirão Preto, que aprovou a realização do projeto. O estudo buscou caracterizar o perfil do profissional que atua nessa área, bem como as atividades previstas, a fim de evidenciar as transformações na política de saúde do município.

O critério de escolha dos entrevistados foi intencional; elegeu-se uma enfermeira em cada um dos cinco Distritos e no nível central, de acordo com o ano de contratação, sendo um profissional por ano de inserção, entre 1988 e 1995 , totalizando 8 enfermeiros. As entrevistas foram agendadas por telefone com antecedência, sendo realizadas pelos bolsistas de iniciação científica no local de trabalho dos profissionais.

Utilizou-se a entrevista semi-estruturada, porque:

“(...)ao mesmo tempo que valoriza a presença do investigador oferece todas as perspectivas para que o informante alcance a liberdade e a espontaneidade necessárias, enriquecendo a investigação" (TRIVIÑOS, 1990).
Após a apresentação dos entrevistadores e dos objetivos da pesquisa, desencadeavam-se três perguntas -chave:

1) O que você veio fazer no Departamento de Vigilância Epidemiológica?

2) O que você faz hoje de diferente do que você fazia antes? A estruturação do Departamento mudou?

3) Houve um pico de contratação de enfermeiros no ano de 1.993. Existe algum acontecimento que justifique esse pico?

As entrevistas foram gravadas; duraram entre vinte e quarenta minutos e, após a sua realização, foram transcritas na íntegra.

\section{TÉCNICA DE ANÁLISE DOS DADOS}

Trata-se de uma abordagem qualitativa, optandose pelo método de análise de conteúdo, proposto por GOMES (1996) Cronologicamente a análise de conteúdo pode abranger as seguintes fases: pré-análise, exploração do material, tratamento dos resultados obtidos e interpretação. Elegeu-se a análise temática que se "encaminha para a contagem de freqüência das unidades de significação como definitórias do caráter do discurso" (MINAYO, 1993).

Inicialmente foi realizada a leitura exaustiva das entrevistas para, em seguida, num processo de aprofundamento horizontal e vertical das mesmas, ir destacando-se as unidades de registro e as temáticas em relevo. (MINAYO, 1993).

Buscou-se através da leitura de cada entrevista revelar os conteúdos manifestos, relacionando-os individualmente às unidades temáticas ao final foi feita uma síntese de todas as entrevistas. Além da unidade temática central emergiu dos dados outra unidade que trata, especificamente, do âmbito da saúde coletiva (investigação epidemiológica; supervisão/coordenação de ações de prevenção e controle; produção de informações).

Quanto à classificação dos dados, para o trabalho operacional de ordenação e separação dos aspectos de análise, utilizou-se o software ETHNOGRAPH 4.0, um processador para pesquisa qualitativa. $\mathrm{O}$ ETHNOGRAPH 4.0 é um gerenciador de dados em pesquisa qualitativa, um programa para gerenciamento $\mathrm{e}$

\footnotetext{
***** A Secretaria Municipal da Saúde assume a completa responsabilidade sobre a gestão da prestação de serviços: planejamento, cadastramento, contratação, controle e pagamento de prestadores ambulatoriais e hospitalares públicos e privados; assume o gerenciamento de toda a Rede Pública existente no município, exceto unidades hospitalares de referência, sob gestão estadual; assume a gestão e o controle das Ações Básicas de Saúde, nutrição e educação, de vigilância epidemiológica, vigilância sanitária e de Saúde do trabalhador no seu território, conforme definido na Comissão Bipartite, recebe mensalmente o total dos recursos financeiros para custeios correspondentes aos tetos ambulatorial e hospitalar estabelecidos (BRASIL, 1993, p.6963)
} 
análise de dados fundamentados no texto. Apresenta um conjunto de recursos que facilitam o controle dos dados, tais como: transcrição de entrevistas, grupos focais, diários de campo, minutos de encontro e outros documentos. Simplifica o processo de categorização, além de busca e escolha dos dados baseados nas categorias de análise do pesquisador.

As mesmas foram emergindo da análise particularizada de cada entrevista, sendo que foi realizada a síntese, relacionando-a com o quadro teórico.

As unidades temáticas inicialmente identificadas foram: O trabalho de enfermagem na Vigilância Epidemiológica; A especificidade do trabalho em Saúde Coletiva; Organização e estrutura do trabalho na Vigilância Epidemiológica; Descentralização das ações de Vigilância Epidemiológica; A integração das ações de saúde e Trabalho interdisciplinar.

Estas temáticas foram reagrupadas para efeito de elaboração teórica e conformadas em uma unidade temática eleita neste estudo: $\Rightarrow O$ trabalho de enfermagem na Vigilância Epidemiológica. Esse tema é resultante das questões teóricas que nortearam a investigação.

\section{DISCUSSÃO E ANÁLISE DOS RESULTADOS}

\section{Unidade Temática Central - O TRABALHO de ENFERMAGEM NA VIGILÂNCIA EPIDEMIOLÓGICA}

A inserção da enfermeira na Vigilância Epidemiológica e as transformações nas políticas de saúde do município

Identificou-se e analisou-se os diferentes períodos em que o profissional enfermeiro foi inserido no Departamento de Vigilância Epidemiológica da Secretaria Municipal de Saúde de Ribeirão Preto, a fim de evidenciar as transformações nas políticas de saúde do município.

Gráfico 1 - Distribuição do número de enfermeiros de acordo com o ano de inserção no Departamento de Vigilância Epidemiológica da Secretaria Municipal de Saúde de Ribeirão Preto, São Paulo

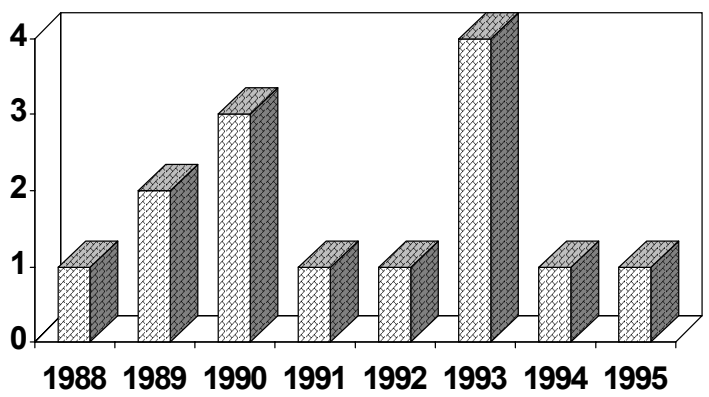

O gráfico 1 mostra que a partir de 1988 os enfermeiros são inseridos no Departamento de Vigilância Epidemiológica e esse número vem crescendo gradativamente, ano a ano, havendo um pico de contratações em 1993.

Quando o Departamento de Vigilância Epidemiológica foi constituído em 1989, as primeiras enfermeiras e profissionais deste serviço executavam todas as ações. Depois ocorre, gradativamente, a divisão de trabalho entre os profissionais.

"Pouco a pouco nós fomos estruturando o serviço, no começo não havia uma divisão de trabalho como tem agora; tem três coordenações dentro do serviço e mesmo quem não tem coordenação fica responsável por determinadas doenças. Esclarecer dúvidas da unidade e do Distrito é responsabilidade do Nível Central. Para cada grupo de doenças tem um profissional responsável, que agrupa os dados, treina o pessoal e organiza o serviço para atender aquele grupo de doenças" (ENT. N. ${ }^{\circ}$ 6).

Ocorre gradativamente a divisão de trabalho na V.E. (nível central) com o aumento do número de profissionais, sendo a implantação dos Programas marcada pela normatização e padronização das ações. Cada conjunto de doenças tem um responsável por sua coordenação, organização, agrupamento de dados, divulgação, fluxo de informações, avaliações e estatísticas, sendo que alguns programas como Tuberculose e Imunização são coordenados por enfermeiros. Observase um processo de descentralização das ações de VE para o nível dos distritos de saúde, a partir de 1993, com predomínio do enfermeiro na composição destas equipes.

Gráfico 2 - Percentual de enfermeiros e sua representatividade em relação aos outros profissionais no Departamento de Vigilância Epidemiológica da Secretaria Municipal de Saúde de Ribeirão Preto - 1996

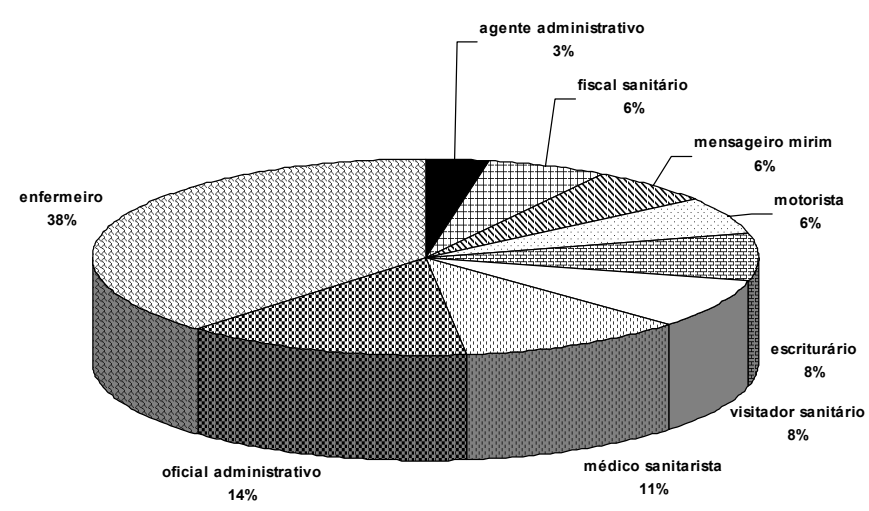


A análise do gráfico mostra a distribuição percentual dos profissionais do Departamento de Vigilância Epidemiológica da Secretaria Municipal de Saúde de Ribeirão Preto, por categoria profissional, no ano de 1996.

Os enfermeiros constituem $38 \%$ do quadro total de profissionais, seguidos por: oficiais administrativos (14\%); médicos sanitaristas (11\%); escriturários e visitadores sanitários (ambos com 8\% cada); fiscais sanitários, motoristas e mensageiros mirins (cada um com $6 \%$ ) e, finalmente, o agente administrativo, com 3\%.

Em 1990/1991 houve no município uma epidemia de dengue. Nesta época tornou-se necessário um maior controle da doença, conseqüentemente, acontece a ampliação com a contratação de recursos humanos.

A municipalização dos serviços de saúde é parte de um processo de descentralização do sistema de saúde, onde novas relações passam a ser estabelecidas entre o nível central e o nível local, entre os diferentes níveis de atendimento, na perspectiva de uma reorganização da atenção à saúde, privilegiando os vínculos dos serviços com os seus usuários, núcleos familiares e comunitários.

"(...)após a epidemia de dengue, a Vigilância Epidemiológica passou a ser muito exigida e conhecida, nós éramos um grupo pequeno, não dávamos conta(...)passando a ser conhecida, ela passa a ser exigida, resultando na necessidade de mais profissionais" (ENT. N. ${ }^{2}$ 2).

A criação de novos distritos (UBDS), cada um com uma equipe da Vigilância Epidemiológica e funcionando 24 horas, acarretou um aumento da demanda de enfermeiros:

"O Departamento tinha como meta aumentar o número de enfermeiros para que ficasse coberto o dia todo, das 7 às 18 horas. Como o enfermeiro faz apenas 4 horas e para nós cobrirmos todos os horários, teve como proposta contratar mais enfermeiros" (ENT. N. ${ }^{\circ}$ 5).

"Nos distritos que tinham enfermeiras num só período passou a ter nos dois; houve um crescimento do número de enfermeiras "(ENT. N. $\left.{ }^{0} 7\right)$.

Vigilância Epidemiológica - Um conhecimento específico

As atividades de Vigilância Epidemiológica requerem conhecimentos específicos de epidemiologia e controle de doenças e agravos à saúde, gerência de programas, supervisão das salas de vacina, requerendo treinamento e formação específica em Saúde Pública.
"A necessidade de reorientar e reorganizar os Serviços de Saúde de forma a atender aos princípios do SUS levou parte dos profissionais de Saúde a procurar, no campo das disciplinas da Epidemiologia, respostas para várias questões postas por esse movimento de mudanças, ou seja, práticas tradicionais sofrendo processos de revisões conceituais e organizacionais" (BARATA, 1992).

A distribuição de enfermeiras, de acordo com sua formação profissional é a seguinte: 9 (nove) enfermeiras fizeram Especialização em Saúde Pública; 2 (duas) em Enfermagem do Trabalho; 1 (uma) em Administração Hospitalar; e 2 (duas) são Mestrandas em Saúde Pública.

$\mathrm{Na}$ atuação do enfermeiro nas Unidades Básicas Distritais de Saúde (UBDS) observa-se através da análise das entrevistas, que todos realizam supervisão das Unidades Básicas de Saúde (UBS) relacionadas à sala de vacina: treinamento de pessoal, controle de reação vacinal e campanhas de vacinação.

"Somos responsáveis pela supervisão de todas as salas de vacina e por todas as doenças de notificação compulsória da nossa área de abrangência, além de fazermos bloqueios e investigações dessas doenças" (ENT. N. ${ }^{\circ}$ 8).

“(...)reação vacinal é algo mais complexo, quem faz as visitas e acompanha o caso é a enfermeira da Vigilância" (ENT. N. $\left.{ }^{\circ} 1\right)$.

Realizam também ações de controle e notificação de doenças que envolvem bloqueio e orientação de exames laboratoriais específicos da VE. A atuação ocorre dentro da área de abrangência da Unidade Básica Distrital de Saúde (UBDS), dependendo do número de Unidades Básicas de Saúde (UBS) da área de abrangência do Distrito.

"A partir da constituição da Vigilância Epidemiológica nós pegamos mais casos, investimos mais, com isso os problemas, a demanda e as unidades cresceram, passando o trabalho a ser diferente, porque a Vigilância passou a estar indo mais de perto" (ENT. N. $\left.{ }^{\circ} 4\right)$.

A relação de trabalho entre as Unidade Básicas de Saúde e a VE é marcada, segundo algumas entrevistas, pelo desconhecimento, falta de preparo e de compromisso dos profissionais de saúde da unidade quando às doenças de notificação compulsória, casos de complicação vacinal.

"Nós temos hoje muitas Unidades de Saúde sem notificar(...)às vezes a pessoa 
não está voltada para as ações de Vigilância Epidemiológica, não consegue entender que o trabalho de prevenção é muito importante" (ENT. N. ${ }^{\circ}$ 4).

Diante do desconhecimento das ações de VE investe-se em treinamento de funcionários para que conheçam sua área de atuação, como também implantouse a informatização que é um instrumento de trabalho que facilita e oferece condições operacionais mais adequadas à organização das atividades e funcionamento da Vigilância Epidemiológica, o que permite acessar dados epidemiológicos e discutir o perfil das doenças de notificação compulsória da área, sendo este um instrumento facilitador do trabalho da enfermeira neste local.

\section{"( )agora vamos informatizar a vacina; a era da informatização da Saúde é um grande avanço" (ENT. N. 1).}

\section{CONCLUSÕES}

Os dados evidenciam que as atividades de Vigilância Epidemiológica desenvolvidas pelo profissional de enfermagem requerem conhecimentos específicos de epidemiologia, controle de doenças e agravos à saúde, gerência de programas (planejamento, avaliação e coordenação), orientação técnica das ações de Vigilância Epidemiológica e vacinação, necessitando o enfermeiro ter formação específica em Saúde Pública.

Estas transformações na política de saúde e, particularmente na Vigilância Epidemiológica, acarretam uma divisão de trabalho entre os agentes, redefinição de papéis (gerência e coordenação de programas) entre nível central e distrital (executores das ações de vigilância).

Verificou-se ainda que existem dificuldades na articulação das ações de Vigilância Epidemiológica entre os níveis central, distrital e local, devido ao desconhecimento e falta de interesse por parte de alguns profissionais, além da complexidade que envolve esse processo de trabalho.

O predomínio do enfermeiro na VE no município se dá pela necessidade de um profissional com conhecimentos mais especializados, formação mais abrangente, desenvolvimento de habilidades técnicas de enfermagem, maior capacitação, conhecimento de medidas de controle (prevenção) para evitar o aparecimento de doenças, trabalho com campanhas, vacinas e imunização, além de haver um predomínio deste profissional nas equipes de VE dos distritos de saúde.

\section{NURSING PROFESSION IN EPIDEMIOLOGIC SURVEILLANCE (ES) AT RIBEIRÃO PRETO} (1988-1996)

The insertion of the nursing profession in Epidemiologic Surveillance (ES) at Ribeirão Preto from 1988 to 1996 has been identified in the process of health care decentralization. The qualitative methodology is used based in bibliographic and documentation sources and by 8 semi-structured interviews. Nurses are in greater number than all other health profissionals and their practice are based on the Epidemiology and management of control side, represented by notifications and visits, and the prevention side, represented by immunization.

KEY WORDS: public health nursing, epidemiologic surveillance

\section{EL ENFERMERO EN LA VIGILANCIA EPIDEMIOLÓGICA EN RIBEIRÃO PRETO (1988-1996)}

El enfermero es considerado en los equipos de vigilancia epidemiológica de la Secretaría Municipal de Salud de Ribeirão Preto en el proceso de municipalización de salud. Con un abordaje cualitativo se obtienen los datos a través de fuentes documentales, bibliográficas y 8 entrevistas semi-estructuradas. El profesional que predomina en el equipo de trabajo es el enfermero, el mismo fundamenta su práctica en la epidemiología y en la gerencia, orientado para el control representado por las notificaciones y visitas y para la prevención representada por la inmunización.

TÉRMINOS CLAVES: enfermería de salud pública vigiláncia epidemiológica

\section{REFERÊNCIAS BIBLIOGRÁFICAS}

01. BARATA, R.B. Reorientação das práticas de V.E. In: SEMINÁRIO NACIONAL DE V.E. Anais. Brasília: MS/FNS/CENEPI/UVE, dez. 1992.
02. BRASIL, Leis etc. Lei N. ${ }^{\circ} 8.080$ de 19 de setembro de 1990. Dispõe sobre as condições para a promoção, proteção e recuperação da Saúde, a organização e o funcionamento dos serviços correspondentes e dá outras providências. Diário Oficial da União, Seção 1, p.18.055-18.059. 
03. CAMPOS, G.W.S. Análise crítica da contribuições da saúde coletiva e a organização das práticas de saúde no SUS. In: FLEURY, S. (org.). Saúde e Democracia: a luta do CEBES. São Paulo: Lemos Editorial, 1997. p.113-124.

04. GOMES, R. A análise de dados em pesquisa qualitativa in MINAYO, M.C.S. (org.). Pesquisa Social: teoria, método e criatividade. 5. ed. Petrópolis: Rio de Janeiro, 1996.

05. MENDES, E.V. Distrito sanitário - O processo social de mudança das práticas sanitárias do Sistema único de Saúde. São Paulo: HUCITEC; Rio de Janeiro: ABRASCO, 1993.

06. MENDES GONÇAVES, R.B. Tecnologia e organização social das práticas de saúde: características tecnológicas do processo de trabalho na rede estadual de centros de saúde de São Paulo. São Paulo, 1986. 416p. Tese (Doutorado) - Faculdade de Medicina da USP.
07. MINAYO, M.C.F. O desafio do conhecimento: pesquisa qualitativa em saúde. 2. ed. São Paulo: Hucitec/ABRASCO, 1993. 269 p.

08. PAIM, J.S. A reorganização das práticas de saúde em distritos sanitários. In: MENDES, E.V. Distrito sanitário: a mudança nas práticas do Sistema Único de Saúde. São Paulo: HUCITEC, 1993. p.187-220.

09. ROUQUAYROL, M.Z.; PONTES. L. A medida da saúde coletiva. In: Epidemiologia e Saúde, 4. ed. Rio de Janeiro: MEDSI, 1994. p.23-76.

10. TRIVIÑOS, A.N.S. Introdução à pesquisa em ciências sociais: a pesquisa qualitativa em educação. São Paulo: Atlantas, 1990. 